\title{
Mejora de la predicción de la resistencia y rigidez de la madera estructural con el método de ultrasonidos combinado con parámetros de clasificación visual
}

\section{Improving the prediction of strength and rigidity of structural timber by combining ultrasound techniques with visual grading parameters}

\author{
M. Conde García(*), J. I. Fernández-Golfín Seco(*) y E. Hermoso Prieto(*)
}

Recepción/Received: 1-XII-06

Aceptación/Accepted: 17-I-07

RESUMEN

Se analiza la posibilidad de aplicar la técnica de transmisión longitudinal de ultrasonidos para la evaluación de la resistencia y módulo de elasticidad a flexión de la madera estructural de las dos especies de mayor interés constructivo y más amplia presencia en obras de rehabilitación: el pino silvestre (Pinus sylvestris L.) y el pino laricio (Pinus nigra Arn.).

Trabajando sobre un total de 1.305 vigas de pino silvestre y 852 de pino laricio se concluye que por sí sola la velocidad de transmisión de ultrasonidos no es un buen predictor ni de la resistencia ni del módulo de elasticidad en flexión, necesitando el complemento de otras variables predictoras.

Se proponen diversos modelos basados en la medición de la velocidad de transmisión de ultrasonidos, de los diámetros relativos del nudo máximo de cara y de canto, de la longitud y de la densidad. Los modelos se proponen tanto a nivel especie como global, comprobándose que es posible emplear un modelo único para ambas especies.

Los modelos propuestos son capaces de explicar entre el 63 y el $73 \%$ de la variabilidad de la resistencia y módulo de elasticidad a flexión.

Palabras clave: ultrasonidos, madera, clasificación visual, propiedades mecánicas, ensayos no destructivos.
SUMMARY

The present study explores the possibility of using longitudinal ultrasound transmission to evaluate the bending strength and modulus of elasticity in structural timber made from the two species most commonly found in Spanish construction and rehabilitation works: Scots pine (Pinus sylvestris L.) and Laricio pine (Pinus nigra Arn.).

An analysis of 1305 Scots pine and 852 Laricio pine beams shows that ultrasound transmission velocity alone can predict neither the bending strength nor the modulus of elasticity and that other predictive variables are required.

A series of models are proposed based on ultrasound transmission velocity measurements, the relative size of the largest face and edge knots, length and density. After running models for each species individually and for the two jointly, a single model is found to be suitable for both.

The models proposed explain from 63 to 73 per cent of bending strength and modulus of elasticity variability.

Keywords: ultrasound, timber, visual grading, mechanical properties, non destructive analysis.

(*) Forest Research Centre (CIFOR-INIA). 


\section{INTRODUCCIÓN}

Los agentes de la degradación de la madera que generan los daños de mayor importancia son los bióticos, es decir, los hongos y los insectos. En ocasiones, estos agentes de la degradación pueden exhibir la particularidad de que sus daños quedan circunscritos al interior de las piezas sin ser visibles al exterior hasta que éstas están altamente afectadas. Esto pasa, por ejemplo, con el ataque por termitas, que sólo es aparente ante ojos expertos siendo visibles en la mayoría de los casos sólo cuando los daños resultan irreparables.

Es necesario también apuntar que muchos de estos agentes degradan mecánica (insectos) o químicamente (hongos) la estructura celular de la madera, lo que genera notables cambios en sus propiedades.

Disponer de una herramienta objetiva y de fácil uso que permita evaluar la capacidad resistente residual de una madera y detectar la presencia o no de tales daños, aunque sean incipientes, es algo fundamental a la hora de poder decidir sobre la conveniencia o no de proceder a la sustitución, mantenimiento o reparación de una estructura en servicio. Herramientas de este tipo permiten ahorrar grandes sumas de dinero al concentrar las labores de reparación en aquellos elementos estructurales que realmente lo necesitan (1).

Es en este campo de la predicción de la resistencia residual de una estructura de madera donde los ultrasonidos, aplicados sobre otros materiales estructurales más habituales en la construcción moderna, como son el hormigón y el acero, se han empleado con cierto nivel de éxito.

Es importante reseñar que paralelamente a los ultrasonidos se están desarrollando para la madera otras técnicas predictivas, que implican el empleo de penetrómetros fractómetros, georradares, etc.

Todos los equipos de transmisión longitudinal de ondas ultrasónicas por la estructura de la madera basan su funcionamiento en la relación existente entre la velocidad de propagación longitudinal de una onda generada en un extremo de la pieza y las propiedades elásticas de la madera, relación que viene expresada mediante la siguiente Ecuación [2]:

Módulo de elasticidad dinámico $=$ Densidad $^{*}$ Velocidad $^{2}$

La velocidad de propagación, así como en ocasiones el nivel de atenuación, de las ondas ultrasónicas inducidas en el material han sido empleadas frecuentemente por diversos autores como variables predictoras de la calidad mecánica del material (2).

\section{INTRODUCTION}

The most damaging type of decay in wood is caused by biotic agents, primarily fungi and insects. Some such agents characteristically cause damage inside pieces only, and as a result the decay is not externally perceptible until the wood is severely damaged. This is the case of termite attack, for instance, which is only visible to the expert eye and usually only after the damage is irreparable.

Moreover, many of these agents occasion mechanical (insects) or chemical (fungi) degeneration of the cellular structure of the timber, prompting major changes in its properties.

An objective, convenient tool is therefore needed to assess the residual strength of timber and detect the presence of even incipient damage, with a view to determining the suitability or otherwise of replacing, maintaining or repairing structures in service. Such a tool would afford considerable savings, for repair and maintenance work could then be focused on the elements actually in need of attention (1).

Ultrasound techniques, used in more common structural materials in modern construction such as concrete and steel, have been applied quite successfully to predict the residual strength of timber structures.

At the same time, however, other predictive instruments are being developed for use with timber, including penetrometers, fractometers, ground penetrating radars and so on.

All ultrasonic wave transmission equipment is based on the relationship between the longitudinal velocity of a wave generated at one end of the piece and the elastic properties of the timber, as expressed by the following Equation [2]:

$$
\text { Modulus of dynamic elasticity = Density } * \text { Velocity }{ }^{2}
$$

The propagation velocity, and on occasion the attenuation level, of the ultrasonic waves induced in wood have been used as variables by a number of authors when predicting its mechanical strength (2). 
La utilidad del método de transmisión de ondas ultrasónicas se basa en el hecho de que la presencia de discontinuidades en la estructura del material, como una grieta o un nudo, produce un aumento del tiempo empleado por la onda para llegar desde el emisor hasta el receptor $y$, por tanto, una disminución en la velocidad, ya que la distancia recorrida por las ondas ultrasónicas es mayor al tener que "sortear" el obstáculo. Una descripción detallada del método queda reflejada en el trabajo de Hermoso et al. (3).

Son varios los estudios llevados a cabo para evaluar la aplicabilidad de los ultrasonidos en la detección de defectos localizados, tales como pudriciones o ataques de termitas (4), la presencia de fendas internas y superficiales producidas tras el secado (5), o la detección de nudos $(6)$ y desviaciones de fibras $(7,8)$. Existen también estudios orientados hacia la estimación de la posición del defecto en el que se inicia el mecanismo de rotura y que determina la tensión de rotura (9), así como hacia el estudio del mecanismo de su propagación (10).

Esta técnica ha sido ampliamente investigada y utilizada en la práctica para la inspección de maderas aserradas en servicio (4,11-17), así como para la caracterización de madera en rollo (18), madera aserrada (19-25) y derivados de la madera (26). Sandoz (27) investigó su uso en la determinación de la resistencia de la madera aserrada estructural de abeto y concluyó sobre la posibilidad de realizar una efectiva clasificación a partir de los datos de las velocidades de propagación. Una interesante revisión del estado del arte puede ser analizada en el trabajo de Beall (28).

Dado que la velocidad de transmisión varía con la estructura del material, para ser utilizable en la práctica el método debe ser evaluado y puesto a punto para cada una de las especies de madera. Por este motivo, el objetivo principal de este trabajo consiste en analizar la aplicabilidad de modelos de predicción de la calidad mecánica de la madera basados en la técnica de transmisión de ultrasonidos y su ajuste a las dos especies de mayor interés constructivo y más amplia presencia en obras de rehabilitación: el pino silvestre y el pino laricio.

\section{MATERIALES Y MÉTODOS}

\subsection{Muestra de ensayo}

Para la realización del presente trabajo se hizo uso de una base de datos del Laboratorio de Estructuras del CIFOR-INIA, que contenía los resultados de 2.157 ensayos sobre piezas de madera estructural de pino silvestre
The principle underlying the ultrasonic wave transmission method is that the presence of discontinuities such as a fissures or knots in the structure of the material lengthens the time taken by the wave to travel from the emitter to the receiver; i.e., the velocity recorded is lower, for the waves must travel a farther distance to "bypass" the obstacle. A detailed description of the method can be found in Hermoso et al. (3).

Studies have been conducted to evaluate the applicability of ultrasound for detecting localized flaws such as rotting or termite attack (4), internal or surface drying fissures (5), knots (6) or slope of grain $(7,8)$. Other surveys have aimed to analyze the propagation mechanism (9) or to estimate the position of the flaw where the failure mechanism originates, as one of the parameters that determines ultimate stress (10).

The technique has been widely researched and used in practice to inspect timber structures in service $(4,11-17)$ as well to characterize small diameter round timber (18), sawn timber (19-25) and wood-based products (26). Studying its use as a way to measure the strength of structural spruce timber, Sandoz (27) drew a series of conclusions about the potential of transmission velocity data for effective grading. See Beall (28) for a comprehensive review of the state-of-the-art.

Since transmission velocity varies with material structure, if it is to be of any practical use, the method must be evaluated and adjusted for each species of timber. The primary aim of the present study, therefore, is to analyze the utility of ultrasound transmission-based models in predicting timber strength and to adjust these models for use with the two most relevant and commonly used species in Spanish construction and renovation work: Scots pine and Laricio pine.

\section{MATERIALS AND METHODS}

\subsection{Test sample}

The information for this study was drawn from the CIFOR-INIA Structures Laboratory database, which contains the results of 2157 tests on structural timber conducted on the occasion of previous projects ${ }^{1}$ (SC00-043, 
y pino laricio, efectuados en diversos proyectos ${ }^{1}$ (SC00-043, SC96-045-C2-2 y AGF99-0176) anteriormente llevados a cabo sobre la caracterización y clasificación de material estructural de la madera de los pinos silvestre (3) y laricio (29).

En el material ensayado se determinaron las velocidades de paso de la onda ultrasónica $\left(\mathrm{V}_{\text {silv }}\right)$, así como, posteriormente, su resistencia y módulo de elasticidad en flexión según UNE-EN 408 (30).

La muestra analizada se compuso de 1.305 piezas de pino silvestre y 852 de pino laricio de varias dimensiones, tal y como puede apreciarse en la Tabla 1. Esta muestra fue en su momento seleccionada por técnicos del Laboratorio de Estructuras del CIFOR-INIA en aserraderos de distintas zonas de procedencia, respetando las exigencias establecidas en las normas UNE-EN 384 (31) y UNEEN 408 (30) de representatividad de la madera de cada especie realmente presente en el mercado. De acuerdo con esto puede afirmarse que la muestra representa fielmente la existente en el mercado.

Todas las probetas tenían en el momento de ensayo un contenido de humedad homogéneo en el intervalo 11-13\%.

\subsection{Medidas ultrasónicas}

Para efectuar las medidas de velocidad de paso de ondas ultrasónicas se empleó un equipo SYLVATEST DUO (C) Concept Bois Structures), dotado de dos palpadores (emisor y receptor) de tipo cónico emitiendo a una frecuencia de $22 \mathrm{kHz}$. Las medidas de la velocidad de paso de la onda entre el emisor y el receptor fueron llevadas a cabo mediante el método directo (palpadores colocados confrontados e insertados en las testas de las piezas).
SC96-045-C2-2 and AGF99-0176) undertaken to characterize and grade Scots (3) and Laricio (29) structural timber.

Ultrasonic wave transmission velocity $\left(V_{\text {silv }}\right)$ and subsequently timber strength and modulus of elasticity were found as stipulated in Spanish and European standard UNE-EN 408 (30).

The test sample comprised 1305 pieces of Scots and 852 pieces of Laricio pine, as shown in Table 1. This sample was gathered by CIFOR-INIA laboratory technicians from sawmills in different areas in accordance with Spanish and European standard UNE-EN 384 (31) and UNE-EN 408 (30) requirements regarding the use of samples representative of timber species actually present on the market.

The moisture content at test time was evenly distributed and ranged from 11 to $13 \%$ in all pieces.

\subsection{Ultrasonic measurements}

Ultrasonic wave velocity was measured on a Concept Bois Structures 22-kHz SYLVATEST DUOC) apparatus, fitted with two conical probes (emitter and receiver). Wave velocity measurements were performed with the direct method (probes directly coupled to opposite ends of the pieces).

Tabla 1 / Table 1

Muestra ensayada

Test sample

\begin{tabular}{|l|c|c|}
\hline $\begin{array}{c}\text { Dimensiones h } \times \text { b } \times \text { I (en mm) } \\
\text { Dimensions } h \times b \times \text { I (in mm) }\end{array}$ & \multicolumn{2}{|c|}{$\begin{array}{c}\text { No piezas } \\
\text { No. of pine }\end{array}$} \\
\hline & $\begin{array}{c}\text { P. laricio } \\
\text { Laricio pine }\end{array}$ & $\begin{array}{c}\text { P. silvestre } \\
\text { Scots pine }\end{array}$ \\
\hline $100 \times 40 \times 2500$ & 115 & 54 \\
\hline $100 \times 50 \times 2500$ & 119 & 93 \\
\hline $150 \times 40 \times 3500$ & 115 & 299 \\
\hline $150 \times 50 \times 3500$ & 122 & 191 \\
\hline $150 \times 70 \times 3500$ & 118 & 214 \\
\hline $200 \times 50 \times 4500$ & 125 & 215 \\
\hline $200 \times 70 \times 4500$ & 138 & 239 \\
\hline TOTAL & 852 & 1305 \\
\hline
\end{tabular}

1. Proyectos del Plan Nacional de $\mathrm{I}+\mathrm{D}+\mathrm{i} /$ National $R+D+I$ Plan projects. 
Para la realización de las medidas con este equipo no se requiere el uso de geles de acoplamiento, ya que el perfecto acoplamiento se consigue por contacto directo con la madera, para lo cual es necesario practicar unos orificios cónicos en la testa de la pieza de $5 \mathrm{~mm}$ de diámetro y $10 \mathrm{~mm}$ de profundidad con el fin de conseguir una superficie de contacto pequeña y adecuada a la forma de los palpadores. La terminación cónica de los palpadores, además, favorece la concentración de energía.

Al igual que para el resto de equipos del laboratorio, el equipo SYLVATEST DUO empleado en las determinaciones fue sometido, de acuerdo con el Manual de Calidad del laboratorio, a comprobación mediante la medición de la velocidad de transmisión de la onda sobre una probeta de un material de referencia suministrado por el propio fabricante del equipo.

\subsection{Ensayo mecánico según UNE-EN 408}

Para llevar a cabo el ensayo mecánico a flexión se siguió fielmente la metodología descrita por la norma UNE-EN 408 (30). El esquema del ensayo queda reflejado en la Figura 1.

Por ensayo, en cada viga se obtuvieron los siguientes datos:

- Tensión de rotura a flexión (MOR) en $\mathrm{N} / \mathrm{mm}^{2}$.

- Módulo de elasticidad global, en $\mathrm{N} / \mathrm{mm}^{2}$ medido en la línea neutra $\left(E_{g}\right)$.

- Densidad $(\rho)$ en $\mathrm{kg} / \mathrm{m}^{3}$.
This apparatus does not require coupling gels; rather, the probes are in direct contact with the timber, intto which two conical holes, $5 \mathrm{~mm}$ in diameter and $10 \mathrm{~mm}$ deep, are drilled -one at each end- to provide a small contact surface suitable for the shape of the sensors. The conical shape of the probes also favours the concentration of energy.

Pursuant to the laboratory's Quality Manual, the SYLVATEST DUO device, like all its other equipment, was calibrated: in this case by measuring the wave transmission velocity through a reference test piece provided by the manufacturer.

\subsection{Bending strength pursuant to UNE-EN 408}

The methodology described in Spanish/European standard UNE-EN 408 (30) was followed to determine timber bending strength. See Figure 1 for a sketch of the test set-up.

The following data were recorded for each piece:

- Ultimate bending stress (MOR) in $\mathrm{N} / \mathrm{mm}^{2}$.

- Overall bending modulus of elasticity in $\mathrm{N} / \mathrm{mm}^{2}$, measured on the neutral axis $\left(E_{g}\right)$.

- Density $(\rho)$ in $\mathrm{kg} / \mathrm{m}^{3}$.

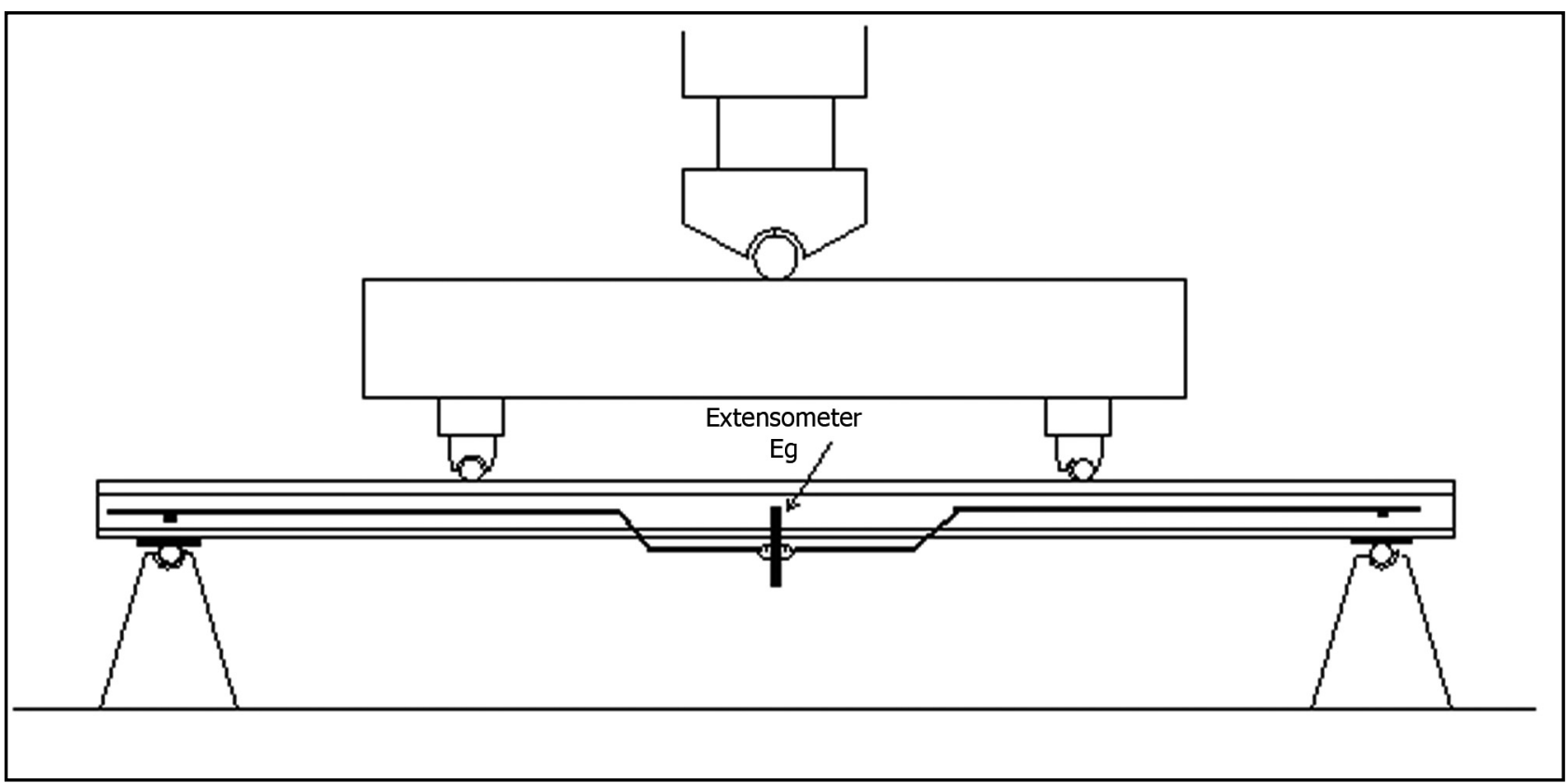

Figura 1. EN 408 test set-up $\left(\mathrm{E}_{\mathrm{g}}\right)$.

Figure 1. EN 408 test set-up $\left(E_{g}\right)$. 
Los ensayos de rotura a flexión se efectuaron en un pórtico de ensayos IBERTEST PELFIB $150 \mathrm{~W}$, dotado con dos células de carga, de 50 y $150 \mathrm{kN}$, así como de dos medidores de desplazamiento LVDT de $20 \mathrm{~mm}$.

Las medidas de masa fueron efectuadas con una balanza METTLER TOLEDO, modelo SB 16001 Delta Range, de 16 $\mathrm{kg}$ de rango de medida y $0,01 \mathrm{~g}$ de precisión, y las de longitud (altura y espesor de la sección transversal) mediante un calibre digital marca Starrett de $200 \mathrm{~mm}$ de rango de medida y 0,01 mm de precisión. Las medidas de longitud de distancia entre apoyos se llevaron a cabo mediante un extensómetro digital MITUTOYO DIGICON TDS551D1 de $5.500 \mathrm{~mm}$ de rango y 0,1mm de resolución.

Para la determinación de la humedad se hizo uso de un xilohigrómetro marca GANN, modelo Hydromette HTR 300.

De acuerdo con la política interna de calidad reflejada en el Manual de Calidad del laboratorio (elaborado de acuerdo con ISO 17025), todos los equipos antes citados son sometidos a calibración externa regular y verificados internamente a intervalos regulares.

\subsection{Clasificación e inspección visual del material}

Todo el material sometido a ensayo fue previamente inspeccionado visualmente de acuerdo con los criterios establecidos en la norma de clasificación visual resistente UNE 56544 (32), que en su redacción sigue los postulados exigidos por las normas UNE-EN 14081-1 (33) y UNE-EN 1310 (34).

Con esta inspección previa se obtuvieron, para cada viga, las siguientes variables:

- $\mathrm{d}_{\mathrm{c}}$ : Diámetro relativo (diámetro dividido entre la anchura de cara de la pieza) del máximo nudo de cara, en \%.

- $d_{\mathrm{h}}$ : Diámetro relativo (diámetro dividido entre el espesor de la pieza) del máximo nudo de canto, en \%.

\subsection{Obtención de los modelos matemáticos de relación entre variables}

Para obtener las relaciones matemáticas entre las distintas variables se aplicaron técnicas de análisis de regresión con modelos lineales. Como variables independientes se emplearon las siguientes:

- Velocidad de transmisión de la onda ultrasónica de cada viga $\left(\mathrm{V}_{\text {silv }}\right)$, expresada en $\mathrm{m} / \mathrm{s}$.

- Diámetro relativo del mayor nudo de cara $\left(\mathrm{d}_{\mathrm{c}}\right)$, expresado en tantos por uno.
A 150-W IBERTEST PELFIB testing machine, fitted with one 50- and one 150-kN load cell and two 20- $\mathrm{mm}$ LVDT transducers, was used to test the pieces to bending failure.

Mass measurements were taken with a METTLER TOLEDO model SB 1600 Delta Range 16-kg scales, with a precision of $0.01 \mathrm{~g}$. Length was measured with a Starett electronic slide calliper (range: $200 \mathrm{~mm}$; resolution: 0.01 $\mathrm{mm}$ ) for cross-section height and thickness, and a MITUTOYO DIGICON TD-S55ID1 digital extensometer (range: $5500 \mathrm{~mm}$; resolution: $0.1 \mathrm{~mm}$ ) for the distance between the supports.

A GANN Hydromette HTR 300 moisture meter was used to measure moisture content.

Pursuant to the laboratory's in-house quality policy, as described in its Quality Manual (formulated to ISO 17025), all the aforementioned equipment is calibrated externally on a regular basis and is subject as well to routine internal verification.

\subsection{Visual grading}

Prior to testing, all samples were visually graded to Spanish standard UNE 56,544 (32), which is at least as demanding as Spanish/European standards UNE-EN 14081-1 (33) and UNE-EN 1310 (34).

The following information was recorded for each piece visually graded:

- $d_{c}$ : Relative diameter (transversal knot diameter divided by the width of the piece) of the maximum knot on the face, in \%.

- $d_{h}$ : Relative diameter (transversal knot diameter divided by the width of the piece) of the maximum knot on the edge, in \%.

\subsection{Mathematical models}

The mathematical relationships among the variables were determined via linear regression analysis. The independent variables used were:

- Ultrasonic wave transmission velocity in each beam $\left(V_{\text {silv }}\right)$, expressed in $\mathrm{m} / \mathrm{s}$.

- Relative diameter of the largest face knot $\left(d_{c}\right)$, expressed as a decimal. 
- Diámetro relativo del mayor nudo de canto $\left(d_{h}\right)$, expresado en tantos por uno.

- Valor de la densidad $(\rho)$, expresado en $\mathrm{kg} / \mathrm{m}^{3}$.

- Longitud de la viga ( $\mathrm{l})$, expresada en $\mathrm{mm}$.

Como variables dependientes se emplearon el valor de tensión de rotura a flexión (MOR) y el del módulo de elasticidad en flexión $\left(E_{g}\right)$, obtenidos por ensayo mecánico según UNE-EN 408 (30), expresados ambos en $\mathrm{N} / \mathrm{mm}^{2}$.

Para determinar tales modelos se empleó el programa informático estadístico STATGRAPHICS 5.0.

\section{RESULTADOS Y DISCUSIÓN}

Obtenidos los valores de ensayo citados en el apartado anterior, se procedió a determinar los parámetros que tenían un efecto significativo en la explicación de la variabilidad de las distintas variables independientes consideradas.

Para analizar la influencia real de cada una de las variables independientes en el modelo predictivo del MOR y
- Relative diameter of the largest edge knot $\left(d_{h}\right)$, expressed as a decimal.

- Density $(\rho)$, in $\mathrm{kg} / \mathrm{m}^{3}$.

- Beam length (I) in $\mathrm{mm}$.

The dependent values were the test values found for ultimate bending strength (MOR) and the bending moduIus of elasticity $\left(E_{g}\right)$, both in $\mathrm{N} / \mathrm{mm}^{2}$, as described in UNE-EN 408 (30).

STATIGRAPHICS 5.0 software was used to formulate the models.

\section{RESULTS AND DISCUSSION}

The aforementioned test values were subsequently used to define the parameters accounting for significant portions of the total variability of the independent variables.

Step-by-step linear regression was performed to analyze the actual effect of each of the independent variables on

Tabla 2 / Table 2

Efecto individual y aditivo de las diversas variables

Individual and combined effects of independent variables on determinants

\begin{tabular}{|c|c|c|}
\hline $\begin{array}{l}\text { Variables dependientes } \\
\text { Dependent variable }\end{array}$ & $\begin{array}{l}\text { Variables independientes } \\
\text { Independent variable }\end{array}$ & $\begin{array}{c}\text { Coef. de determinación } \\
\text { Determinant }\end{array}$ \\
\hline \multirow{6}{*}{ MOR } & $\mathrm{V}_{\text {silv }}$ & 0.385 \\
\hline & Edyn & 0.284 \\
\hline & $d_{c}$ & 0.211 \\
\hline & $d_{h}$ & 0.314 \\
\hline & $\rho$ & 0.151 \\
\hline & 1 & 0.036 \\
\hline \multirow{6}{*}{$E_{g}$} & $\mathrm{~V}_{\text {silv }}$ & 0.522 \\
\hline & $E_{d y n}$ & 0.353 \\
\hline & $d_{c}$ & 0.105 \\
\hline & $d_{h}$ & 0.165 \\
\hline & $\rho$ & 0.227 \\
\hline & 1 & 0.017 \\
\hline \multirow{5}{*}{ MOR } & $\mathrm{V}_{\text {silv }}$ & 0.385 \\
\hline & $v_{\text {silv }}+d_{h}$ & 0.531 \\
\hline & $v_{\text {silv }}+d_{h}+d_{c}$ & 0.546 \\
\hline & $v_{\text {silv }}+d_{h}+d_{c}+\rho$ & 0.609 \\
\hline & $v_{\text {silv }}+d_{h}+d_{c}+\rho+1$ & 0.648 \\
\hline \multirow{5}{*}{$E_{g}$} & $\mathrm{~V}_{\text {silv }}$ & 0.522 \\
\hline & $V_{\text {silv }}+\rho$ & 0.687 \\
\hline & $\mathrm{V}_{\text {silv }}+\rho+\mathrm{d}_{\mathrm{h}}$ & 0.705 \\
\hline & $\mathrm{V}_{\text {silv }}+\rho+\mathrm{d}_{\mathrm{h}}+\mathrm{d}_{\mathrm{c}}$ & $0.705^{*}$ \\
\hline & $\mathrm{V}_{\text {silv }}+\rho+d_{h}+1$ & $0.705 *$ \\
\hline
\end{tabular}

Efecto no significativo / Effect not statiscally significant. 
$E_{g}$ se llevó a cabo un ajuste, por regresión y paso a paso, de modelos lineales. La Tabla 2 recoge los valores de los coeficientes de determinación obtenidos mediante modelos lineales con una sola variable independiente, así como el efecto que tiene la adición de las diversas variables independientes.

De acuerdo con los datos de la Tabla 2, se observa que la velocidad de transmisión longitudinal de la onda $\left(V_{\text {silv }}\right)$ no es por sí misma capaz de predecir con suficiente precisión ni la resistencia (MOR) ni el comportamiento elástico $\left(E_{g}\right)$ a flexión de la madera estructural. Por dicho motivo, tal y como se aprecia en la Tabla 2, será necesario complementar la medida de velocidad de onda con una cuidadosa inspección de la estructura incorporando variables adicionales basadas en mediciones cualitativas $\left(d_{h}\right.$ y $\left.d_{c}\right)$ y físicas ( $\mathrm{y}$ r) efectuadas sobre el material a evaluar.

Respecto al empleo de la variable $V_{\text {silv }}$ o de su derivada $E_{d y n}$ (obtenida mediante la expresión $\left.\mathrm{E}_{\text {dyn }}=\rho^{*}\left(\mathrm{~V}_{\text {silv }}\right)^{2}\right)$, la Tabla 2 recoge mejores coeficientes de determinación empleando la primera $\left(\mathrm{V}_{\text {silv }}\right)$. Por dicho motivo en la Tabla 3 no figura $E_{d y n}$ como variable independiente, sino $V_{\text {silv. }}$. Este resultado se observó tanto en la predicción de la resistencia (MOR), como en la del módulo de elasticidad $\left(E_{g}\right)$.

De acuerdo con la información de la Tabla 2, en la Tabla 3 figuran los modelos más significativos empleados para la predicción tanto de la resistencia (MOR), como del módulo de elasticidad en flexión $\left(E_{g}\right)$. En dicha tabla figuran los modelos obtenidos tanto para cada una de las dos especies consideradas, como para el conjunto de las dos.

Todos los modelos incluidos en la Tabla 3, así como sus diversas variables independientes, se ha comprobado que tienen un efecto significativo en la predicción del valor de las distintas variables dependientes consideradas. the model used to predict MOR and $E_{g}$. Table 2 gives the values of the determinants found from linear models with a single independent variable, and the variations in those values when the remaining independent variables were included, one at a time.

The data in Table 2 show that wave longitudinal transmission velocity $\left(V_{\text {silv }}\right)$ alone did not predict the bending strength or elastic behaviour $\left(E_{g}\right)$ of structural timber with sufficient precision. Hence, velocity measurements must be supplemented with minute inspection of the structure and the inclusion of additional qualitative $\left(d_{h}\right.$ and $d_{c}$ ) and physical ( $I$ and r) measurements.

Likewise further to Table 2, better determinants were obtained for both strength (MOR) and modulus of elasticity $\left(E_{g}\right)$ with the variable $V_{\text {silv }}$ than with its derivative $E_{d y n}$ (found from the expression $\left.E_{d y n}=\rho^{*}\left(V_{\text {silv }}\right)^{2}\right)$. Consequently, only the former is shown as an independent variable in Table 3.

In keeping with the information in Table 2, Table 3 lists the most relevant models used for predicting strength (MOR) and bending modulus of elasticity $\left(E_{g}\right)$. The table shows the models derived for each of the two species separately and for the two together.

All the models in Table 3, as well as their independent variables, proved to have a significant effect on the prediction of the values of the dependent variables considered.

Tabla 3 / Table 3

Modelos

Models

\begin{tabular}{|c|c|c|c|c|c|c|c|c|}
\hline \multicolumn{9}{|c|}{$Y=A+B^{*} V_{\text {silv }}+C^{*} d c+D^{*} d_{h}+E^{*} I+F^{*} \rho-$ Error } \\
\hline \multicolumn{9}{|c|}{ Variables independientes / Independent variable } \\
\hline Var. Dep. & A & B & C & D & $\mathbf{E}$ & $\mathbf{F}$ & Error & $\mathbf{R}^{\mathbf{2}}$ \\
\hline \multicolumn{9}{|c|}{ Pino silvestre / Scots pine } \\
\hline MOR & -51.8232 & 0.0156779 & -17.027 & -19.5393 & -0.00207201 & 0.0744176 & 10.7 & 0.632 \\
\hline $\mathbf{E}_{\mathbf{g}}$ & -12465.8 & 3.16324 & NS & -1009.53 & 0.208585 & 13.8258 & 1250.5 & 0.698 \\
\hline \multicolumn{9}{|c|}{ Pino laricio / Laricio pine } \\
\hline MOR & -63.5851 & 0.0203009 & -13.3613 & -21.5019 & -0.0081278 & 0.0915088 & 12.0 & 0.695 \\
\hline$E_{g}$ & -16568.4 & 4.16592 & NS & -1242.14 & -0.19773 & 14.9703 & 1460.8 & 0.732 \\
\hline \multicolumn{9}{|c|}{ Ambas especies / Laricio\&Scots pine } \\
\hline MOR & -50.0276 & 0.016988 & -16.7471 & -21.3532 & -0.00454516 & 0.0772907 & 11.4 & 0.648 \\
\hline$E_{g}$ & -13355.0 & 3.50204 & NS & -1242.65 & NS & 13.9503 & 1367.4 & 0.705 \\
\hline
\end{tabular}

NS: No significativo / Not significant. 
Del análisis de los datos de la Tabla 3 se deduce que, dados los valores obtenidos en los coeficientes de determinación de los distintos modelos, la técnica de transmisión de ultrasonidos resulta ligeramente más eficaz en la predicción del comportamiento elástico de la madera que en la predicción de la resistencia.

Si se incorpora el error de predicción en el modelo la predicción de los valores individuales, resulta muy segura ya que en tan sólo el $2 \%$ de los casos se sobrestima el valor del MOR en más de $10 \mathrm{~N} / \mathrm{mm}^{2}$ respecto del MOR real y el del $\mathrm{E}_{\mathrm{g}}$ en más de $1000 \mathrm{~N} / \mathrm{mm}^{2}$.

De acuerdo con la información de las Tablas 2 y 3, debe hacerse constar el hecho de que la evaluación del tamaño relativo del nudo de canto $\left(d_{h}\right)$ tiene un efecto significativo en los modelos, cualquiera que sea la variable dependiente a predecir $\left(M O R, E_{g}\right)$, mientras que el tamaño relativo del nudo de cara $\left(\mathrm{d}_{\mathrm{c}}\right)$ sólo tiene efecto significativo en la predicción de la resistencia a flexión (MOR). Este distinto comportamiento puede atribuirse al diferente efecto que las desviaciones locales de fibras, debidas a la presencia de los nudos (en la cara o en el canto), producen en la resistencia y elasticidad de la madera sometida a esfuerzos de flexión.

Como se puede apreciar, la adición de la densidad mejora notablemente los modelos si bien añade una complicación práctica en obra, ya que la medición de esta variable es sumamente complicada, motivo por lo cual deberán ser empleados métodos de estimación (penetrómetros) o de sondeo (barrenas Pressler).

El efecto de la longitud en algunos de los modelos sugiere un problema de atenuación de la onda con la longitud del elemento a medir, ya que la longitud es una variable que implícitamente forma parte de la medida de la velocidad de transmisión de la onda.

La comparación entre sí de los distintos modelos obtenidos para cada especie y para el global de las dos permite llevar a la conclusión de que, sin merma significativa en la calidad de la predicción, es posible emplear un único modelo para ambas especies, lo que simplificará enormemente su empleo en obra, ya que las dos especies consideradas, el pino silvestre (Pinus sylvestris) y el pino laricio (Pinus nigra), siendo muy comunes en obras de rehabilitación en España, son en la práctica indistinguibles anatómicamente.

\section{CONCLUSIONES}

Las principales conclusiones que es posible obtener del presente trabajo son las siguientes:
An analysis of the data in Table 3 shows that, in light of the values obtained for the determinants in the different models, ultrasound transmission predicted elastic behaviour slightly more accurately than strength in timber.

When error prediction was incorporated into the model, the prediction of individual values was highly reliable, inasmuch as MOR was overestimated by more than 10 $\mathrm{N} / \mathrm{mm}^{2}$, and the value of $E_{g}$ by over $1000 \mathrm{~N} / \mathrm{mm}^{2}$ in only $2 \%$ of the cases.

The relative size of the edge knot $\left(d_{h}\right)$ had a significant effect on the models (see Tables 2 and 3), regardless of the dependent variable to be predicted $\left(M O R, E_{g}\right)$, whereas the relative size of the face knot $\left(d_{c}\right)$ significantly affected the prediction of ultimate bending strength (MOR) only. This differential behaviour may be attributed to the varying effect of local slope of grain, due to the presence of knots (on the face or on the edge), on timber strength and elasticity when the material is subjected to bending stress.

While the inclusion of density improved the models substantially, it also involved certain practical complexities, for this parameter is not readily measured in situ, but must be estimated with penetrometers or probes (Pressler borer).

In some of the models, the effect of length is suggestive of wave attenuation with the length of the piece, since length is an implicit component of wave transmission velocity.

The conclusion drawn from a comparison of the various models obtained for each species and the overall model for both is that a single model can be used for the two species without significantly jeopardizing the quality of the prediction. This simplifies the procedure immensely in construction works, for the two species considered, Scots pine (Pinus sylvestris) and Laricio pine (Pinus nigra), both commonly used in rehabilitation work in Spain, are anatomically indistinguishable in practice.

\section{CONCLUSIONS}

The main conclusions to be drawn from the above results are as follows: 
- La velocidad de transmisión longitudinal de la onda no es por sí misma capaz de predecir con suficiente precisión ni la resistencia ni el comportamiento elástico a flexión de la madera estructural. Puede obtenerse una buena predicción empleando junto con $V_{\text {silv }}$ variables independientes adicionales, tales como el diámetro relativo del nudo de cara, el diámetro relativo del nudo de canto, la longitud de la viga y la densidad de la madera.

- Aun cuando en el presente trabajo se proponen modelos específicos para cada una de las especies consideradas, se ha comprobado que es posible emplear modelos comunes para la predicción del MOR, $E_{g}$ de ambas especies.

- El empleo de los modelos propuestos en la predicción de valores individuales resulta seguro, ya que las sobrestimas se limitan a tan sólo el $2 \%$ de los casos, con sobrestimaciones no superiores a $10 \mathrm{~N} / \mathrm{mm}^{2}$ en el MOR y a $1.000 \mathrm{~N} / \mathrm{mm}^{2}$ en $E_{g}$.

\section{AGRADECIMIENTOS}

Los autores quieren expresar su agradecimiento al Dr. Díez Barra por la revisión de los originales.
- Longitudinal wave transmission velocity alone does not provide sufficiently precise predictions of bending strength or elastic behaviour in structural timber. More accurate predictions can be obtained when this parameter is used jointly with other independent variables, such as the relative diameters of the face and edge knots, the length of the beam and timber density.

- While the study proposes individual models for each species considered, a single model has been shown to be suitable for predicting MOR and $E_{g}$ in both.

- The models proposed can be safely used for predicting individual values, given that overestimates, which accounted for only $2 \%$ of the cases, were no greater than $10 \mathrm{~N} / \mathrm{mm}^{2}$ for MOR or $1000 \mathrm{~N} / \mathrm{mm}^{2}$ for $E_{g}$.

\section{ACKNOWLEDGEMENTS}

The authors wish to thank Dr. Díez Barra for revising the original manuscript.

\section{BIBLIOGRAFÍA / BIBLIOGRAPHY}

(1) Brashaw, B. K.; Vatalaro, R.; Wacker, J. y Ross, R.: "Condition Assessment of Timber Bridges: part 2. Evaluation of Several StressWave Timer Devices", Gen. Tech. Report FPL-GTR-160. Madison, WI : U.S. Department of Agriculture, Forest Service, Forest Products Laboratory, 2005, 12 pp.

(2) Pellerin, R. y Ross, R.: Nondestructive evaluation of wood. USDA Forest Service, Forest Products Laboratory, Madison WI. Forest Products Society. ISBN 1-892529-26-2, 2002, 209 pp.

(3) Hermoso Prieto, E.: Caracterización mecánica de la madera estructural de Pinus sylvestris L. Tesis Doctoral. Universidad Politécnica de Madrid (UPM), España, 2001.

(4) Fujii, Y.: "Using acoustic emission monitoring to detect termite activity in wood", Forest Prod. Journal, vol. 40, n 1 (1990), pp. 34-36.

(5) Fuller, J. J.; Ross, R. J. y Dramm, J. R.: Honeycomb and surface check detection using ultrasonic nondestructive evaluation. Res. Note FPL-RN-0261. Madison, WI: US. Department of Agriculture, Forest Service, Forest Products Laboratory, 1994, 6 pp.

(6) Karsulovic, J. T.; León, L. A.; Gaete, L.: "Ultrasonic detection of knots and annual ring orientation in Pinus radiata lumber", Wood and Fiber Science, 32 (3) (2000), pp. 278-286.

(7) Bucur, V.: "Relationship between grain angle of wood specimens and ultrasonic velocity", Catgut Acoustical Society Inc., 41 (1984), pp. 30-35.

(8) Kabir, M. F.: "Prediction of ultrasonic properties from grain angle", Journal of the Institute of Wood Science, vol. 15, n 5 (89) (2001),

pp. 235-246.

(9) Sandoz, J. L.; Benoit, Y. y Demay, L.: "Wood testing using acousto-ultrasonic", $12^{\text {th }}$ International Symposium on Nondestructive Testing of Wood, 2000, pp. 97-104.

(10) Reiterer, A.; Stanzl-Tschegg, E. y Tschegg, K.: 2000. "Mode I fracture and acoustic emission of softwood and hardwood", Wood Science and Technology, 34 (2000), pp. 417-430.

(11) Beall, F. C.; Tiitta, M. y Biernacki, J. M.: "The use of acousto-ultrasonics to detect biodeterioration in structural wooden members", Proceedings of the Nondestructive Testing and Evaluation of Infraestructure, vol. 2 (1998), Am. Soc. for Nondestructive Testing, Columbus, OH, pp. 181-206.

(12) Németh, L.: "Evaluation of built-in timbers by non-destructive testing", $12^{\text {th }}$ International Symposium on Nondestructive Testing of Wood, 2000, pp. 457-461. 
Mejora de la predicción de la resistencia y rigidez de la madera estructural con el método de ultrasonidos combinado con parámetros de clasificación visual Improving the prediction of strength and rigidity of structural timber by combining ultrasound techniques with visual grading parameters

(13) Clausen, A.; Ross, R. J.; Forsman, J. W.; Balachowski, J. D.: "Condition assessment of roof trusses of Quincy Mine Blacksmith Shop in Keweenaw National Historical Park", USDA Forest Service, Forest Products Laboratory. Research Note FPL-RN-0281, 2001, 4 pp.

(14) Ross, R. J. y Hunt, M. O.: Stress wave timing non-destructive evaluation tools for inspecting historic structures. A guide for use and interpretacion, Gen. Tech. Rep. FPL-GTR-119. Madison, WI: US. Department of Agriculture, Forest Service, Forest Products Laboratory, 2000, $15 \mathrm{pp}$.

(15) Ross, R. J.; Pellerin, R. F.; Forsman J. W.; Erickson, J. R. y Lavinder, J. A.: Relationship between stress wave transmission time and compressive properties of timbers removed from service.

(16) Esteban, M.: Determinación de la capacidad resistente de la madera estructural de gran escuadría y su aplicación en estructuras existentes de madera de coníferas. Tesis Doctoral. Universidad Politécnica de Madrid (UPM), España, 2003.

(17) Arriaga, F.; Esteban, M. y Relea, E.: "Evaluation of the load carrying capacity of large cross section coniferous timber in standing structures", Mater. Construcc., vol. 55 (280) (2005), pp. 43-52.

(18) Wang, X.; Ross, R. J.; Mattson, J. A.; Erickson, J. R.; Forsman, J. W.; Geske, E. A.; Wehr, M. A.: Several nondestructive evaluation techniques for assessing stiffness and MOE of small-diameter logs. Res. Paper FPL-RP-600. Madison, WI: US. Department of Agriculture, Forest Service, Forest Products Laboratory, 2001, $12 \mathrm{pp}$.

(19) Arriaga, F.; İ́niguez, G. y Esteban, M.: "Assessment of strength and stiffness properties using longitudinal stress wave on structural gross cross section timber of radiata pine", 14th International Symposium on Nondestructive Testing of Wood. Eberswalde, Germany, 2005. ISBN 3-8322-3949-9.

(20) Arriaga, F.; Íñiguez, G.; Esteban, M. y Fernández-Golfín, J. I.: "Structural Tali timber (Erythrophleum ivorense A. Chef., Erythrophleum suavolens Brenan.): Assesment of strength and stiffness properties using visual and ultrasonic methods", Holz als Roh und Werkstoff, 64 (2006), pp. 357-362.

(21) Fernández-Golfín, J. I.; Díez, M. R. y Hermoso, E.: "Relationships between grade determining properties of Spanish Scots and Laricio pine structural timber", Mater. Construcc., 270 (53) (2003), pp. 45-55.

(22) Hermoso, E; Fernández-Golfín, J. I. y Díez, M. R.: "Evaluación de la clasificación resistente de la madera estructural mediante ultrasonidos", $10^{\circ}$ Congreso Nacional de Ensayos No Destructivos, Cartagena, España, actas, 2003, pp. 187-195.

(23) Machado, J. S.; Sardinha, R. y Cruz, H.: "Evaluation of lengthwise variation of mechanical properties by ultrasounds", Proceedings of the $5^{\text {th }}$ Conference on Timber Engineering, Montreaux (Switzerland), vol. 2 (1998), pp. 304-311.

(24) Kessel, M. H.; Plinke, B.; Augustin, R.; Huse, M.: "Strength grading of construction timber with large cross sections", Proceedings of the 5th Conference on Timber Engineering, Montreaux (Switzerland), vol. 1 (1998), pp. 557-562.

(25) Kuklik, P. y Dolejs, J.: "Nondestructive evaluation of structural timber", Proceedings of the $5^{\text {th }}$ Conference on Timber Engineering, Montreaux (Switzerland), vol. 1 (1998), pp. 692-699.

(26) Kazemi Najafi, S. y Ebrahimi, Gh.: "Three methods for the prediction of longitudinal ultrasonic wave velocity in particleboard and fiberboard", Proceedings of the 14th International Symposium on Nondestructive Testing of Wood. Eberswalde, Germany, 2005, ISBN 38322-3949-9.

(28) Beall, F. C.: "Overview of the use of ultrasonic technologies in research on wood properties", Wood Sci. and Technol., 36 (2002), pp. 197-212.

(27) Sandoz, J. L.: "Grading of construction timber by ultrasound", Wood Science and Technology, 23 (1989), pp. 95-108.

(29) Conde García, M.: Caracterización de la madera estructural de Pinus nigra Subsp. Salzmannii. Tesis Doctoral. Universidad Politécnica de Madrid (UPM), España, 2005.

(30) UNE-EN 408 (30):2004: Timber structures. Structural timber and glued laminated timber. Determination of some physical and mechanical properties.

(31) UNE-EN 384 (29):2004: Structural timber. Determination of characteristic values of mechanical properties and density.

(32) UNE 56.544:2003: Visual grading for structural sawn timber. Coniferous timber.

(33) UNE-EN 14081-1 (33):2005. Timber structures. Strength graded structural timber with rectangular cross section. Part 1: General requirements.

(34) UNE-EN 1310 (32):1997. Madera aserrada y en rollo. Método de medida de las singularidades.

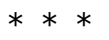

